\title{
DISTRIBUIÇÃO DO BENEFíCIO DE PRESTAÇÃO CONTINUADA ENTRE AS REGIÕES BRASILEIRAS
}

\author{
DISTRIBUTION OF THE CONTINUED PAYMENT BENEFITS AMONG BRAZILIAN \\ REGIONS
}

\author{
Ely Mitie Massuda ${ }^{1}$ \\ Edson Luciano Rudey ${ }^{2}$ \\ Marcelo Picinin Bernuci ${ }^{3}$ \\ Sonia Maria Marques Gomes Bertolini ${ }^{4}$ \\ Mateus Dias Antunes 5 \\ Fabrício Hiroiuki Oda ${ }^{6}$ \\ Mirian Ueda Yamaguchi ${ }^{7}$
}

Resumo: Este estudo apresenta a análise da distribuição das concessões do Benefício de Prestação Continuada entre as regiões brasileiras nos 20 anos desde sua implementação. A pesquisa é um estudo descritivo, cujos dados foram obtidos no Ministério do Desenvolvimento Social e Combate à Fome. As concessões aumentaram em todas as regiões brasileiras ao longo do período, apresentando declínio no ritmo de crescimento com decalagem temporal entre as regiões. O maior número de concessões, em termos absolutos, ocorreu para as regiões mais populosas do país, Sudeste e Nordeste. O Nordeste apresentou a maior taxa de concessão para as pessoas com deficiência, e quanto aos idosos, as maiores taxas foram observadas no Norte, regiões que apresentam indicadores socioeconômicos inferiores. Os resultados indicaram um perfil distinto das concessões nas regiões brasileiras sugerindo a necessidade de se considerar suas diferenças para o enfrentamento das inequidades apontadas na presente pesquisa.

Palavras-chave: assistência social; pobreza; política social; vulnerabilidade social.

Abstract: This study aimed to analysis the distribution pattern of the Continued Payment Benefits (CPB) concessions within the different Brazilians regions in the 20 years of its implementation. This is a descriptive study whose data was obtained in the portal of the Brazilian Ministry of Social Development and Fight Against Hunger. Concessions increased in all Brazilian regions throughout the period, showing a decline in the rate of growth with a temporal breakdown between regions. The largest number of concessions, in absolute terms, occurs for the most populous regions of the country, Southeast and Northeast. The Northeast region presented the highest concession rate followed by the North, Midwest, South and Southeast regions for people with disabilities. Concerning the elderly, the highest rates were observed in the North and CentralWest regions, followed by the Northeast Southeast and South regions. The results indicated a distinct profile of the concessions in the Brazilian regions suggesting the need to consider their differences to address the inequities pointed out in the present research.

Keywords: social assistance; poverty; public policy; social vulnerability.

\footnotetext{
1 Docente do Programa de Pós-Graduação em Promoção da Saúde (PPGPS) e do Programa em Gestão do conhecimento nas Organizações (PPGGCO) do Centro Universitário de Maringá (UNICESUMAR), Brasil. E-mail: elymitie.m@gmail.com.

2 Doutorando pela Faculdade de Medicina da Universidade do Porto/Instituto Nacional de Previdência Social (INSS), Maringá/PR, Brasil. E-mail: edsonrudey@hotmail.com.

3 Docente do Programa de Pós-Graduação em Promoção da Saúde (PPGPS) e do Programa em Gestão do conhecimento nas Organizações (PPGGCO) do Centro Universitário de Maringá (UNICESUMAR), Brasil. E-mail: mbernuci@gmail.com.

4 Docente do Programa de Pós-Graduação em Promoção da Saúde (PPGPS) e do Programa em Gestão do conhecimento nas Organizações (PPGGCO) do Centro Universitário de Maringá (UNICESUMAR), Brasil. E-mail: sonia.bertolini@unicesumar.edu.br.

5 Mestre em Promoção da Saúde, Faculdade Unyleya, Brasília/DF, Brasil. E-mail: mateus_antunes03@hotmail.com.

6 Pós-doutorando no Programa de Pós-Graduação em Promoção da Saúde (PPGPS) do Centro Universitário de Maringá (UNICESUMAR), Brasil. E-mail: fabricio_oda@hotmail.com.

7 Docente do Programa de Pós-Graduação em Promoção da Saúde (PPGPS) do Centro Universitário de Maringá (UNICESUMAR), Brasil. E-mail: mirianueda@gmail.com.
} 


\section{INTRODUÇÂO}

Os impactos dos riscos ambientais sobre a saúde se acentuam e representam uma das principais causas de morte, doença e incapacidade entre as populações pobres e vulneráveis, especialmente nos países em desenvolvimento (WHO, 2018). No cenário global de transformações demográficas e ambientais, a garantia de renda e o acesso aos serviços de saúde às populações vulneráveis, configuram uma das mais urgentes preocupações das políticas públicas sociais.

A vulnerabilidade e a velhice são reconhecidas pela Constituição Federal do Brasil de 1988 para proteção social (BRASIL, 1988). Pessoas com deficiência e idosos têm direito a receber, desde 1996, o Benefício de Prestação Continuada (BPC), uma transferência incondicional de renda no valor de um salário-mínimo mensal, configurando-se como o maior programa de transferência direta de renda, em termos de volume de recursos no país (BRASIL, 1998; BRASIL, 1995; BRASIL, 2007). É um dos mais importantes mecanismos de proteção social às pessoas que se encontram em situação de dupla vulnerabilidade dada a condição de extrema pobreza e idade avançada e/ou presença de deficiência mental ou física (DUARTE et al., 2017; JACOUD; MESQUITA; PAIVA, 2017). Trata-se de um benefício assistencial e não previdenciário, portanto, não contributivo.

O BPC integra o Sistema Único de Assistência Social (SUAS) e é operacionalizado pelo Instituto Nacional do Seguro Social (INSS) sob a tutela do Ministério do Desenvolvimento Social e Combate à Fome (MDS). Possuem direito ao benefício, pessoas que tenham mais de 65 anos e pessoas com deficiência que comprovem renda mensal bruta familiar per capita abaixo de 1/4 do salário mínimo vigente. Para acesso ao benefício, pessoas com deficiência devem, obrigatoriamente, passar por avaliação médica pericial e social em que se analisam fatores ambientais, de atividades e participação, e as funções do corpo. O idoso deve comprovar a idade e a condição de renda por meio de documento legal (BRASIL, 2015).

Nas últimas décadas, mudanças sociais importantes ocorreram no Brasil caracterizadas pela redução da pobreza e desigualdade. Embora essa redução tenha ocorrido no país como um todo, existem diferenças entre as regiões nesse processo, de forma que aquelas mais suscetíveis aos problemas socioeconômicos foram as menos beneficiadas (ANDRADE et al., 2013). Apesar de importantes para amenizar a extrema pobreza, políticas de transferência de renda para indivíduos como o BPC não reduziram as disparidades existentes entre as regiões do país (ARAÚJO; FLORES, 2017). Embora a extrema pobreza venha se reduzindo no Brasil, as diferenças entre as regiões ainda persistem (FURSTENAU; WINK JUNIOR, 2016).

Se consideradas as desigualdades existentes no país, o benefício poderia se consolidar em uma política pública de enfrentamento das desigualdades no país e entre as regiões de modo a favorecer a equidade. 
Em consonância com a urgência de reflexões sobre a necessidade de reavaliação da política assistencial coberta pelo BPC, o presente estudo objetivou analisar o padrão da distribuição das concessões nas diferentes regiões do país, desde sua implementação até o ano de 2015, uma vez que o Brasil não apresenta uniformidade em seu território, no que se refere aos aspectos sociais, econômicos ou ambientais.

\section{MÉTODO}

O estudo descritivo teve como base dados secundários do Ministério do Desenvolvimento Social e Combate à Fome, acessados no sítio http://www.mds.gov.br/relcrys/bpc/download_beneficiarios_bpc.htm. Os dados referemse aos BPC da Assistência Social concedidos às pessoas com deficiência (B87) e aos idosos (B88), em diferentes regiões do Brasil, por meio do número de concessões no período de 1996 a 2015.

As regiões do Brasil consideradas no estudo foram: Norte (Rondônia, Acre, Amazonas, Roraima, Pará, Amapá e Tocantins), Nordeste (Maranhão, Piauí, Ceará, Rio Grande do Norte, Paraíba, Pernambuco, Alagoas, Sergipe e Bahia), Centro-Oeste (Mato Grosso do Sul, Mato Grosso, Goiás e Distrito Federal), Sudeste (Minas Gerais, Espírito Santo, Rio de Janeiro e São Paulo) e Sul (Paraná, Santa Catarina e Rio Grande do Sul).

As taxas de concessão do BPC foram calculadas utilizando a seguinte equação:

\section{Taxa de concessões $=\frac{\text { número de benefícios no mês de dezembro }}{\text { número de indivíduos na população de interesse }} \quad x 1000$}

As taxas indicam o número de benefícios concedidos a cada mil indivíduos pertencentes à população de interesse (pessoas com deficiência ou idosos), na determinada região e no mês de dezembro do ano considerado.

Conforme as idades mínimas de concessão dos benefícios para os idosos foram realizados ajustes de acordo com as implementações das leis vigentes. Sendo assim, para cálculo da estimativa da taxa de concessões para os idosos, a população considerada foi a de indivíduos com idade igual ou superior a 70 anos para os anos de 1996 e 1997, com idade igual ou superior a 67 anos para os anos de 1998 a 2003 e com idade igual ou superior a 65 anos para os anos de 1999 a 2015, em cada uma das regiões do país. As informações sobre a distribuição da população são disponibilizadas por categorias de idade pelo Instituto Brasileiro de Geografia e Estatística (IBGE) no sítio http://www.ibge.gov.br/home/estatistica/populacao/projecao_da_populacao/2013/. Uma vez que tal distribuição da população por categorias de idade não se encontra disponível para as regiões do país em anos anteriores a 2000, tais quantidades foram 
estimadas considerando a proporção de indivíduos em cada categoria, no ano 2000 , multiplicada pela população total dos anos em questão. Para os beneficiários da categoria pessoas com deficiência considerou-se a população total de cada região no cálculo da taxa de concessões, obtidas no IBGE no sítio http://www.ibge.gov.br/home/.

Para sintetizar as modificações ocorridas na taxa de concessões no mês de dezembro a cada ano para as duas categorias (pessoas com deficiência e idosos), calcularam-se os números-índices relativos para cada categoria de beneficiários, que indicam a variação na taxa entre dois anos consecutivos. O número-índice do primeiro ano do período considerado é fixado como 100:

$$
\text { Número índice }=\frac{\text { taxa de concessões no ano } i}{\text { taxa de concessões no ano } i-1} \quad x 100
$$

Os dados e as informações presentes nos referidos bancos de dados foram incluídos em uma base de dados no software Excel e abrangeram as seguintes variáveis: BPC para os idosos, BPC para as pessoas com deficiência, mês e ano do benefício e região do Brasil. A análise descritiva para o cálculo das taxas e obtenção de gráficos foi realizada com o programa estatístico R versão 3.2.2.

Para comparar o comportamento das taxas de concessões entre as regiões do Brasil, ajustou-se um modelo de regressão linear:

$$
\operatorname{Taxa}_{i}=\beta_{0}+\beta_{1} \operatorname{Ano}_{i}+\varepsilon_{i}
$$

em que $i=1996, \ldots, 2015$ e $\varepsilon \mathrm{N}(0$,$) , para cada região, calculando os respectivos$ intervalos de $95 \%$ de confiança para o parâmetro de cada região. A inclinação da reta de regressão $\left(\boldsymbol{\beta}_{1}\right)$ indica o aumento na taxa de concessões ao longo do tempo, uma vez que representa a variação na taxa a cada ano acrescido.

Do mesmo modo, para investigar possíveis diferenças entre as taxas médias de concessões, foram calculadas as médias das taxas para cada região e seus respectivos intervalos de confiança comparados. Calculadas as estimativas e intervalos de confiança para a inclinação da regressão e média de cada região, para ambas as categorias de beneficiários, os intervalos foram comparados a fim de identificar diferenças significativas entre os parâmetros de cada região. Assim, intervalos sobrepostos, identificados por letras iguais, indicam que não há diferença estatística significativa da inclinação (ou média) entre duas regiões.

A pesquisa foi aprovada pelo Comitê de Ética do Centro Universitário de Maringá (UniCesumar) conforme parecer número 1.918.380.

\section{RESULTADOS}

Em números absolutos, as concessões aumentaram em todas as regiões brasileiras ao longo do período analisado, para ambas as categorias, pessoas com 
deficiência e idosos, atingindo um total de 4.242.697 benefícios em 2015. Desse total, 2.323.794 benefícios foram concedidos a pessoas com deficiência, sendo 30,53\% para a região Sudeste, $39,76 \%$ para a Nordeste, $11,39 \%$ para a Sul, $10,51 \%$ para a Norte e $7,81 \%$ para a Centro-Oeste. Quanto aos demais, 1.918 .903 benefícios dirigiram-se para idosos, dos quais, 39,44\%, 32,10\%, 9,76\%, 9,64\% e 9,05\% para as regiões brasileiras, na mesma ordem, respectivamente.

Os dados referentes aos benefícios concedidos a cada mil pessoas com deficiência, e os da trajetória das concessões por meio dos números-índices estão apresentados na Figura 1. Nota-se que para os anos 1996, 2010 e 2015, o padrão de concessão dos benefícios para cada mil pessoas mostrou-se heterogênea. Em 1996, a ordem de regiões que receberam mais concessões por mil pessoas foi: Nordeste $(3,51)$, Centro-Oeste $(2,37)$, Norte $(2,27)$; Sudeste $(1,07)$ e Sul $(1,04)$. A partir de 2004 , a região Sul ultrapassou a região Sudeste de forma que, em 2010, apresenta-se a seguinte ordem: Nordeste $(12,9)$, Norte $(11,38)$, Centro-Oeste $(9,80)$, Sul $(7,32)$ e Sudeste $(6,62)$. Em 2015 permanece o mesmo arranjo, porém, com elevação nas taxas: Nordeste $(16,34)$, Norte $(13,98)$, Centro-Oeste $(11,74)$, Sul $(9,06)$ e Sudeste $(8,27)$. Com relação à evolução dos números-índices, observam-se oscilações ao longo do período. O ritmo de crescimento das taxas de concessão para as pessoas com deficiência, se mostra em declínio a partir de 2011, para a região Sul, 2012 para as regiões Centro-Oeste, Nordeste e Norte e 2013 para a região Sudeste. 
Figura 1 - Taxas de concessões do BPC e números-índices relativos das taxas de concessões de BPC para pessoas com deficiência, de acordo com a região do Brasil, de 1996 a 2015.
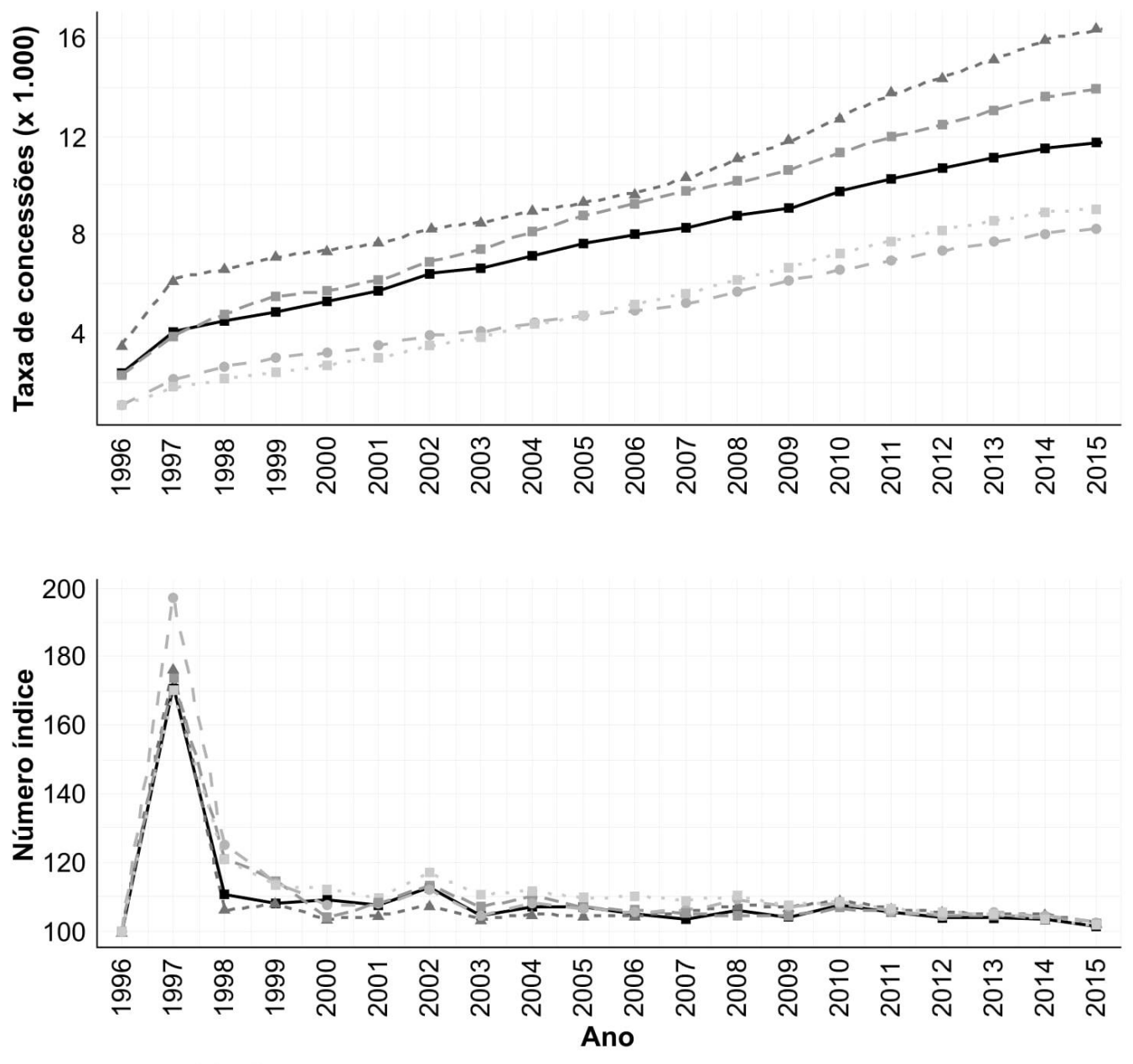

Região: $\rightarrow$ - Centro-Oeste $-\Delta-$ Nordeste $=-$ Norte $\rightarrow$ - Sudeste $-=$ Sul

Fonte: BRASIL (2017). Elaborado pelos autores.

A Figura 2 apresenta os dados referentes às taxas de concessões de BPC (por mil pessoas) para idosos. Em 1996, observa-se o seguinte comportamento: região Centro-Oeste $(17,85)$, Norte $(15,58)$, Nordeste $(9,75)$, Sudeste $(5,35)$ e Sul $(3,81)$. Em 2010, as posições se invertem de forma que o Norte se coloca em primeiro lugar $(242,38)$, Centro-Oeste em segundo lugar $(205,38)$, seguidos pelo Nordeste $(151,69)$, Sudeste $(101,09)$ e Sul $(73,37)$. Em 2015, a ordenação se mantém: Norte $(234,33)$, Centro-Oeste $(175,28)$, Nordeste $(154,32)$, Sudeste $(98,66)$ e Sul $(69,37)$.

Em 1997 em relação a 1996, de acordo com os números-índices, ainda no que se refere aos idosos, as taxas de concessões do benefício aumentaram em 81,54\%, $119,85 \%, 100,98 \%, 108,47 \%$ e $94,43 \%$ nas regiões Centro-Oeste, Nordeste, Norte, Sudeste e Sul, respectivamente. Em 2004, em relação ao ano anterior, os aumentos foram de menor amplitude, de $41,29 \%, 33,14 \%, 34,25 \%, 36,78 \%, 39,90 \%$ para as mesmas regiões, na mesma ordem. A partir desse período, verificou-se redução dos 
índices de forma que atingiram patamares inferiores ao ano anterior: em 2010 no Centro-Oeste; em 2011 no Sul; em 2012 no Norte; 2013 no Sudeste; em 2015 no Nordeste. Ou seja, a partir desses anos, nessas regiões, as taxas de concessão do BPC para idosos decaíram comparativamente ao ano precedente.

Figura 2 - Taxas de concessões de BPC e números-índices relativos das taxas de concessões de BPC para pessoas idosas, de acordo com a região do Brasil, de 1996 a 2015.
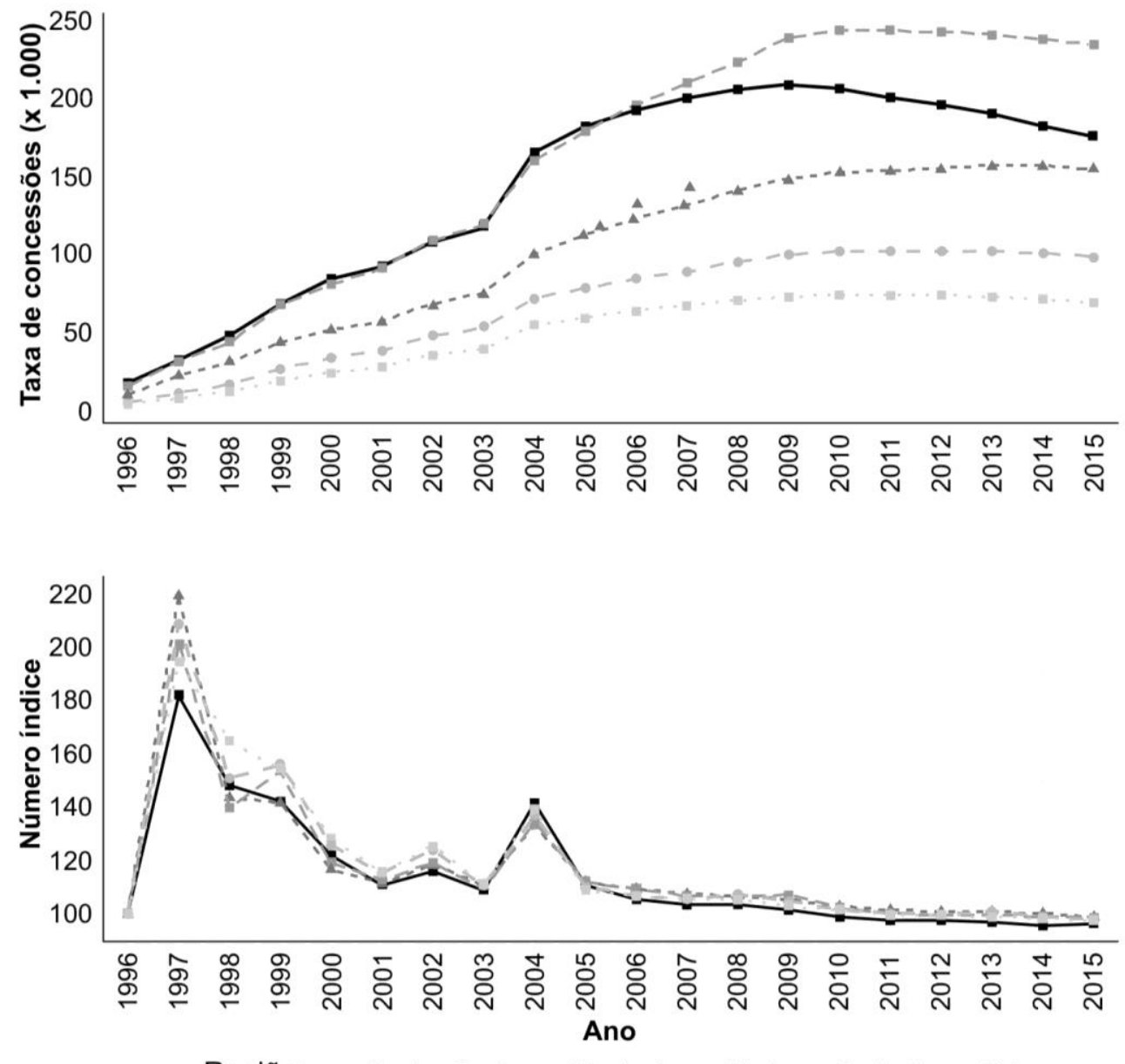

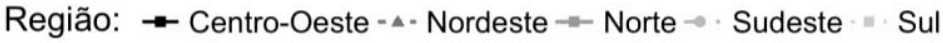

Fonte: BRASIL (2017). Elaborado pelos autores.

A Tabela 1 apresenta os dados referentes à comparação da inclinação da regressão e média da taxa de concessões de BPC para pessoas com deficiência entre as regiões do Brasil, de 1996 a 2015. As regiões Norte e Nordeste apresentaram aumento na taxa de concessões anuais para pessoas com deficiência, sendo que as inclinações destas regiões não diferiram significativamente entre si. A região Sudeste apresentou a menor variação no crescimento da taxa de concessões e o comportamento de sua inclinação foi diferente das outras regiões.

As regiões Nordeste, Norte e Centro Oeste tiveram as maiores médias gerais da 
taxa de concessões no período de 1996 a 2015, mas não diferiram significativamente entre si. As regiões Sul e Sudeste apresentaram as menores taxas médias de concessões, e não diferiram significativamente.

Tabela 1 - Comparação da inclinação da regressão e média da taxa de concessões de BPC para idosos entre as regiões do Brasil, de 1996 a 2015.

\begin{tabular}{l|ccc|ccc}
\hline \multirow{2}{*}{ Região } & \multicolumn{3}{c|}{ Inclinação $\left(\boldsymbol{\beta}_{\mathbf{1}}\right)$} & \multicolumn{3}{c}{ Média $(\boldsymbol{\mu})$} \\
& Estimativa & $\boldsymbol{I} \boldsymbol{C}\left(\boldsymbol{\beta}_{\mathbf{1}}, \mathbf{9 5} \%\right)$ & Estimativa & $\boldsymbol{I} \boldsymbol{C}(\boldsymbol{\mu}, \mathbf{9 5} \%)$ \\
\hline Norte & 13,25 & $(11,30 ; 15,20)$ & a & 159,62 & $(121,35 ; 197,89)$ & a \\
Centro-Oeste & 9,69 & $(7,09 ; 12,30)$ & ab & 143,16 & $(112,62 ; 173,71)$ & a \\
Nordeste & 8,52 & $(7,45 ; 9,58)$ & b & 101,56 & $(77,24 ; 125,88)$ & ab \\
Sudeste & 5,63 & $(4,75 ; 6,52)$ & C & 67,55 & $(51,18 ; 83,92)$ & bc \\
Sul & 4,02 & $(3,28 ; 4,76)$ & C & 49,31 & $(37,43 ; 61,19)$ & c \\
\hline \multicolumn{5}{c}{ * Letras iguais indicam não haver diferença significativa. } \\
\end{tabular}

Os dados referentes à comparação da inclinação da regressão e média da taxa de concessões de BPC para idosos entre as regiões do Brasil, de 1996 a 2015, estão apresentados na Tabela 2. A região Norte apresentou a maior inclinação e média para a taxa de concessões de BPC para idosos, indicando que o aumento da taxa de concessões a cada ano, assim como a taxa média do período são maiores para esta região. A inclinação da região Norte não diferiu significativamente da apresentada pela região Centro Oeste. Também não houve diferença significativa entre as médias das regiões Norte, Centro Oeste e Nordeste.

Por outro lado, tanto para a inclinação da regressão quanto para a média da taxa de concessões, não há indícios de que exista diferença significativa entre as regiões Sudeste e Sul, que apresentaram as menores estimativas dos parâmetros.

Tabela 2 - Comparação da inclinação da regressão e média da taxa de concessões de BPC para pessoas portadoras de deficiência entre as regiões do Brasil, de 1996 a 2015.

\begin{tabular}{l|ccc|ccc}
\hline \multicolumn{1}{c}{ Região } & \multicolumn{3}{|c|}{ Inclinação $\left(\boldsymbol{\beta}_{\mathbf{1}}\right)$} & \multicolumn{3}{c}{ Média $(\boldsymbol{\mu})$} \\
& Estimativa & $\boldsymbol{I} \boldsymbol{C}\left(\boldsymbol{\beta}_{\mathbf{1}}, \mathbf{9 5} \%\right)$ & Estimativa & IC $(\boldsymbol{\mu}, \mathbf{9 5} \%)$ \\
\hline Nordeste & 0,60 & $(0,55 ; 0,65)$ & a & 10,25 & $(8,57 ; 11,94)$ & a \\
Norte & 0,58 & $(0,55 ; 0,60)$ & a & 8,82 & $(7,22 ; 10,42)$ & a \\
Centro-Oeste & 0,46 & $(0,43 ; 0,48)$ & b & 7,72 & $(6,44 ; 8,99)$ & a \\
Sul & 0,44 & $(0,42 ; 0,45)$ & b & 5,17 & $(3,96 ; 6,38)$ & b \\
Sudeste & 0,35 & $(0,33 ; 0,37)$ & c & 4,99 & $(4,01 ; 5,97)$ & b \\
\hline \multicolumn{5}{c}{ * Letras iguais indicam não haver diferença significativa. } \\
& Fonte: BRASIL (2017). Elaborado pelos autores
\end{tabular}




\section{DISCUSSÃO}

Com intuito de prover informações sobre o perfil das concessões do BPC ao longo da trajetória da implantação dessa política, avaliou-se a distribuição das concessões nas diferentes regiões do país. Os dados compilados mostram que o número de concessões para pessoas com deficiência e idosos aumentou ao longo do período, em termos absolutos, em todas as regiões. Na distribuição entre as regiões, a maior parcela da categoria de pessoas com deficiência coube ao Nordeste, diferentemente da categoria idosos que abriga na região Sudeste o maior número absoluto de concessões.

As taxas de BPC para pessoas com deficiência aumentaram continuamente em todas as regiões. $O$ fato decorre também dos aspectos legais de elegibilidade. $O$ Decreto $n^{\circ} 1.744 / 1995$ (BRASIL, 1995) conferia a elegibilidade de pessoas com deficiência ao BPC, aquelas portadoras de lesões ou anomalias irreversíveis que provocassem incapacidade permanente (BRASIL, 2007). Em decorrência da Convenção Internacional dos Direitos das Pessoas de 2007, Decreto 6.214/2007 que regulamenta o BPC à pessoa com deficiência e aos idosos e Decreto 6949/2009 (BRASIL, 2009) que promulga a Convenção Internacional sobre os Direitos das Pessoas com Deficiência, a deficiência passa a ser reconhecida como limitações infligidas pelo meio físico e pela cultura ao desempenho da autonomia e independência de pessoas com limitações de natureza física (ANDRADE et al., 2013; BRASIL, 2009; BRASIL 2007). A caracterização da pessoa com deficiência passa a considerar aquelas que são portadoras de incapacidade devido a doenças e aquelas portadoras de deficiência, ou seja, integrando a compreensão de saúde nos aspectos biológicos, sociais e individuais. A partir de 2007, adotou-se a Classificação Internacional de Funcionalidade (CIF) para a avaliação de pessoas com deficiência (BRASIL, 2007).

Observando-se as taxas dos benefícios concedidos para pessoas com deficiência, verificou-se que as maiores são observadas no Nordeste, seguido pelo Norte e CentroOeste. Sudeste e Sul inverteram suas posições em 2005, quando a primeira ultrapassa as taxas verificadas na segunda. Destaca-se que o Nordeste surge em primeiro lugar para o número absoluto de concessões que, assim como o Norte, apresentaram também as maiores inclinações da regressão $(\beta 1)$ e média da taxa de concessões de BPC $(\mu)$. O agrupamento de regiões de saúde no Brasil por meio da tipologia baseada no Índice de Desenvolvimento Humano (IDH) classificou o Nordeste e o Norte como regiões com baixos e muitos baixos índices de longevidade, renda e escolaridade, ao passo que o Sul, Centro Oeste e Sudeste registraram índices alto e muito alto em sua maior parte (DUARTE et al., 2015).

O processo de análise das pessoas com deficiência para a concessão do BPC consiste na avaliação médico-pericial e avaliação social englobando funções do corpo, fatores ambientais (barreiras para execução de atividades e participação social). Problemas na elegibilidade da pessoa deficiente quanto ao direito do benefício têm sido discutidos devido aos elevados índices de indeferimento, resultando na grande 
demanda de processos de judicialização, além da heterogeneidade na avaliação das pessoas com deficiência apesar da adoção da CIF (FARIAS; BUCHALLA, 2005; BRASIL, 2007; MACÊDO; OLIVEIRA, 2015; COSTA et al., 2016).

Apesar do crescimento do número de concessões para pessoas com deficiência, identificaram-se barreiras sistemáticas de acesso ao benefício devido às avaliações da condição social e médica, resultando em elevadas taxas de indeferimento (COSTA et al., 2016). Nesse contexto, a judicialização dos processos se retrata na quantidade de benefícios concedidos por essa via atingindo $29,58 \%$ do total dos benefícios da categoria em 2015 no país. Ao mesmo tempo, observa-se que as concessões judiciais sobre o total vêm se elevando em todas as regiões, destacando-se que as maiores variações aconteceram no Nordeste brasileiro (BRASIL, 2016).

No que se refere ao BPC para os idosos, em que pese o esperado aumento das concessões no período que segue sua implantação, verificou-se um movimento ascendente das taxas até 2004 em todas as regiões, observando-se uma inflexão mais acentuada entre 2003 e 2004. A primeira mudança ocorreu em 1998 devido a aprovação da Lei 9.720 em novembro daquele ano (BRASIL, 1998), reduzindo a idade mínima de 70 anos para 67 anos. Em 2004, começa a vigorar o Estatuto do Idoso (BRASIL, 2003) diminuindo a idade mínima para 65 anos e, além disso, a existência de um idoso beneficiário do BPC na unidade familiar (esposa/esposo; companheiro/companheira; filhos/filhas, menores de 21 anos ou inválidos; irmã/irmãos, menores de 21 ou inválidos; pai/mãe) deixa de ser considerado no cálculo de renda (a regra não foi estendida para pessoas com deficiência). Associam-se aos fatos, o envelhecimento populacional ampliando o número de idosos e consequente chegada à idade permitida de acesso ao benefício de uma geração submetida a instabilidades no mercado de trabalho durante a vida ativa (ANSILIERO, 2005; PENALVA; DINIZ; MEDEIROS, 2010).

As concessões do BPC para os idosos, considerando taxas para cada mil habitantes, diferem substancialmente do que se apresenta em termos absolutos. Embora a maior taxa de concessões do BPC, inicialmente, tenha se registrado na região Centro-Oeste, o Norte assume a posição de liderança ao longo do período, seguido do Centro-Oeste. O comportamento evidencia-se na comparação entre as regiões, quando o Norte mostrou a maior inclinação da regressão $(\beta 1)$ indicando maior velocidade no crescimento das taxas de concessão, seguido pelo Centro-Oeste, embora sem diferença significativa. Da mesma forma, em relação à média da taxa $(\mu)$ de concessões, o mesmo padrão no que se refere à classificação das regiões foi verificado, observandose as primeiras posições para o Norte e o Centro-Oeste.

Chama a atenção a relação inversa entre as concessões de BPC para idosos e o número proporcional de pessoas com 65 anos ou mais (IBGE, 2017), visto que nas regiões Norte e Centro-Oeste, essa população representa 6,51\% e 7,95\% do total, respectivamente, e são as duas regiões que apresentam as maiores taxas de concessão, enquanto que, no Sudeste, $9,88 \%$ da população são idosos e no Sul 
$10,83 \%$, e são as duas regiões que demandam menores taxas de BPC.

Constata-se aumento da judicialização do BPC para idosos no país, verificandose nas regiões Sul e Centro Oeste maior percentual do benefício por essa via (BRASIL, 2016; XIMENES, 2016). O aumento da judicialização ocorre em razão da demanda pelo benefício de pessoas que não se encaixam ou ultrapassam os limites da elegibilidade (SILVEIRA et al., 2016).

A taxa de crescimento do número de concessões para idosos foi superior à das pessoas com deficiência até 2008, a partir do qual a situação se inverte, pois, a quantidade de concessões ao segundo grupo permanece superior ao do primeiro grupo até o final da série histórica apresentada no presente estudo (BRASIL, 2016).

A posição de liderança da região Norte nas concessões do BPC para idosos e do Nordeste para as pessoas com deficiência, sugere vínculo com o Índice de Desenvolvimento Humano (IDH). Em 2010, o IDH da região Nordeste foi de 0,660; Norte, 0,684; Centro-Oeste, 0,753; Sudeste, 0,754 e Sul, 0,756(18) (BRASIL, 2012). Portanto, o IDH que sintetiza educação, longevidade e Produto Interno Bruto per capita como fatores determinantes para o desenvolvimento e qualidade vida da população de uma região, associados aos resultados desta pesquisa, assinalam que as regiões com menor IDH abrigam maior número de pessoas que atendem aos critérios de inclusão ao BPC.

Esse quadro também espelha o analfabetismo funcional que tem no Nordeste a maior participação dentre as regiões, de $17,4 \%$ da população em 2012. Da mesma forma, é possível compreender a posição da região Norte que possui $10 \%$ da população na mesma condição. No Sudeste e no Sul, 4,8\% e 4,4\% da população, respectivamente, foram considerados analfabetos funcionais (BRASIL, 2012).

Em se tratando de renda domiciliar per capita, o Nordeste apresenta o menor provento, de $\mathrm{R} \$ 774,25$ em 2016 . Em seguida, apresenta-se o Norte com $\mathrm{R} \$ 874,43$ no mesmo ano, o Sudeste com renda de $R \$ 1.250,00$, o Sul cuja renda foi de $R \$ 1.470,00$ e, finalmente, o Centro-Oeste que ostenta a maior renda domiciliar per capita de $\mathrm{R} \$ 1.478,25$ (IBGE, 2017).

O fato é aparentemente contraditório, quando se observa que o Centro-Oeste surge como a segunda região com maior taxa de concessão do BPC para idosos e terceira no que se refere ao benefício para pessoas com deficiência. No entanto, apesar do PIB per capita apresentar-se elevado em algumas regiões, a situação de necessidade de amparo por parcela maior ou menor da população se manifesta na distribuição de renda. Embora o Centro-Oeste indique a maior renda per capita no Brasil, ocupa a terceira posição no número no que diz respeito à população com renda menor do que $1 / 4$ do salário mínimo em 2012 , pois $5,2 \%$ dos habitantes dessa região vivem nessa condição. Nas regiões Nordeste e Norte, esse percentual chega a 20,9\% e 18,4\%, respectivamente. O Sudeste e o Sul atingem o patamar de 4,8\% e 4,4\%, nessa ordem, 
no mesmo ano (BRASIL, 2018). O índice de Gini demonstra que as regiões Norte $(0,6319)$ e Nordeste $(0,6277)$ apresentaram as maiores desigualdades, a região Centro Oeste indica índice intermediário $(0,6019)$, abaixo do Sudeste $(0,585)$ e Sul $(0,5337)$ (CASTRO et al., 2010).

As desigualdades também se revelam quando se observa que, entre 1995 e 2008, período considerado de estabilidade econômica no Brasil, houve redução das taxas de pobreza, mas não ocorreu de modo uniforme entre as regiões brasileiras. Nesse período, na região Sul a taxa de pobreza absoluta (rendimento médio domiciliar per capita de até meio salário mínimo mensal) foi reduzida em $47,1 \%$ e de extrema pobreza (rendimento médio domiciliar per capita de até um quarto de salário mínimo mensal) em 59,6\%; na região Sudeste, em 34,8\% e 41,0\%; na região Nordeste, em 28,8\% e 40,4\%; na região Centro-Oeste, em 12,7\% e 33,7\%; e em região Norte, de $14,9 \%$ e 22,8\%, respectivamente (CASTRO et al., 2010). Portanto, evidenciam as regiões Norte e Centro Oeste com as menores reduções da pobreza absoluta e extrema pobreza. Sendo assim, as maiores quedas das taxas de pobreza, absoluta ou extrema, aconteceram nas regiões que exibem as menores taxas de concessão de BPC.

Portanto, ao se analisar as taxas de concessões do BPC para pessoas com deficiência e idosos, confirmou-se que as maiores foram registradas naquelas regiões em que os indicadores refletem as desigualdades sociais e econômicas.

\section{CONCLUSÃO}

O número de concessões do BPC para pessoas com deficiência e para idosos aumentou continuamente ao longo dos 20 anos da assistência, tendo reduzido o ritmo de crescimento nos últimos anos. Na perspectiva do padrão de distribuição das concessões nas regiões brasileiras, para ambas as categorias do BPC, constatou-se dessemelhança quando comparadas às taxas de concessão por mil habitantes e as concessões em termos absolutos. As regiões mais populosas, Sudeste e Nordeste, sobressaem-se em números absolutos. Em se tratando de taxas, a região Nordeste, no caso das pessoas com deficiência e a região Norte, no que se refere aos idosos, atingem maiores índices. As discussões sobre a concessão do BPC e a questão da elegibilidade de população vulnerável devem levar em consideração as características sociais, econômicas, ambientais e as disparidades regionais existentes no país. Embora se tenha identificado perfil distinto das concessões nas regiões brasileiras, ainda há necessidade de estudos que considerem o efeito dos benefícios no enfrentamento das inequidades regionais brasileiras.

\section{AGRADECIMENTOS}

Ao Instituto Cesumar de Ciência, Tecnologia e Inovação - ICETI, Conselho 
Nacional de Desenvolvimento Científico e Tecnológico - CNPq e Coordenação de Aperfeiçoamento de Pessoal de Nível Superior - CAPES pela concessão de bolsas de auxílio pesquisa.

\section{REFERÊNCIAS}

ANDRADE, M. V. et al. Desigualdade socioeconômica no acesso aos serviços de saúde no Brasil: um estudo comparativo entre as regiões brasileiras em 1998 e 2008. Economia Aplicada, v. 17, n. 4, p. 623-645, 2013.

ANSILIERO, G. Evolução na concessão e emissão de benefícios assistenciais de prestação continuada. Informe da Previdência Social, v. 18, n. 10, p. 1-20, 2005.

ARAÚJO, V.; FLORES, P. Redistribuição de renda, pobreza e desigualdade territorial no Brasil. Revista de Sociologia e Política, v. 25, n. 63, p. 159-182, 2017. Disponível em: <http://www.redalyc.org/html/238/23852993007/>. Acesso em: 08 jun. 2018.

BRASIL. Constituição da República Federativa do Brasil. Brasília, DF: Diário Oficial da União, 5 out. 1988.

BRASIL. Cartilha: Benefício de Prestação Continuada da Assistência Social. Ministério do Desenvolvimento Social e Combate à Fome. Brasília, DF. 2015

BRASIL. Decreto no 1.744, de 8 de Dezembro de 1995. Regulamenta o benefício de prestação continuada devido à pessoa portadora de deficiência e ao idoso, de que trata a Lei $n^{\circ} 8.742$, de 7 de dezembro de 1993, e dá outras providências. Diário Oficial da União Brasília, DF, 1995. Disponível em: <http://www.planalto.gov.br/ccivil_03/decreto/antigos/d1744.htm>. Acesso em: 30 abr. 2018.

BRASIL. Decreto no 6.214, de 26 de Setembro de 2007. Regulamenta o benefício de prestação continuada da assistência social devido à pessoa com deficiência e ao idoso de que trata a Lei no 8.742, de 7 de Dezembro de 1993, e a Lei no 10.741, de 10 de Outubro de 2007. Diário Oficial da União. Brasília, DF, 2007. Disponível em: <http://www.planalto.gov.br/ccivil_03/_ato2007-2010/2007/decreto/d6214.htm>. Acesso em: 30 abr. 2018.

BRASIL. Ministério do Desenvolvimento Social e Combate à Fome (MDS). Benefício de Prestação Continuada da Assistência Social: Quantitativo de benefícios e recursos investidos por unidade da federação de pagamento no período de 1996 a 2017. 2017. Disponível em:

<http://www.mds.gov.br/relcrys/bpc/download_beneficiarios_bpc.htm>. Acesso em: 30 abr. 2018.

BRASIL. Decreto n 6.949 , de 25 de agosto de 2009. Promulga a Convenção Internacional sobre os Direitos das Pessoas com Deficiência e seu Protocolo Facultativo, assinados em Nova York, em 30 de março de 2007. Diário Oficial da União. Brasília, DF, 2009. Disponível em:

$<$ http://www2.camara.leg.br/legin/fed/decret/2009/decreto-6949-25-agosto-2009590871-publicacaooriginal-115983-pe.html>. Acesso em: 08 jun. 2018.

BRASIL. Ministério do Desenvolvimento Social e Combate à Fome (MDS), Secretaria 
Nacional de Assistência Social (SNAS), Departamento de Benefícios Assistenciais (DBA). Nota Técnica sobre as concessões judiciais do Benefício de Prestação Continuada da Assistência Social - BPC e sobre o processo de judicialização do mesmo. Nota Técnica n. 03/2016. Brasília, DF: MDS/SNAS/DBA, 2016.

BRASIL. Lei no 9.720, de 30 de Novembro de 1998. Dá nova redação a dispositivos da Lei no 8.742, de 7 de dezembro de 1993, que dispõe sobre a organização da Assistência Social, e dá outras providências. Diário Oficial da União, Brasília, DF, 1998. Disponível em: <http://www.planalto.gov.br/CCivil_03/leis/L9720.htm>. Acesso em: 30 abr. 2018.

BRASIL. Lei no 10.741, de 10 de Outubro de 2003. Dispõe sobre o Estatuto do Idoso e dá outras providências. Brasília: Diário Oficial da União, Brasília, DF, 2003.

Disponível em: <http://www.planalto.gov.br/ccivil_03/leis/2003/l10.741.htm>. Acesso em: 30 abr. 2018.

BRASIL. Ministério da Saúde (MS). DATASUS (TABNET). Informações de Saúde. Indicadores e Dados Básicos (IDB) referentes ao ano de 2012. Brasília, DF: Ministério da Saúde, 2018. Disponível em: <http://tabnet.datasus.gov.br/cgi/idb2012/a12tb.htm>. Acesso em: 30 abr. 2018.

BRASIL. Ministério da Saúde (MS). DATASUS (TABNET). Informações de Saúde. Proporção de pessoas com baixa renda - Brasil, 2018. Disponível em: <http://tabnet.datasus.gov.br/cgi/tabcgi.exe?ibge/censo/cnv/pobrezauf.def>. Acesso em: 30 abr. 2018.

BRASIL. Ministério do Desenvolvimento Social e Combate à Fome (MDS). Avaliação das pessoas com deficiência para acesso ao Benefício de Prestação Continuada da assistência social: um novo instrumento baseado na Classificação Internacional de Funcionalidade, Incapacidade e Saúde. Brasília, DF: MDS/MPS, 2007.

CASTRO, J. A. et al. Dimensão, evolução e projeção da pobreza por região e por estado no Brasil. Comunicados Ipea, n. 58, 2010. Disponível em:

<http://www.ipea.gov.br/agencia/images/stories/PDFs/100713_comuni58pobreza.pdf>. Acesso em: 30 abr. 2018.

COSTA, N. R. et al. Proteção social e pessoa com deficiência no Brasil. Ciência \& Saúde Coletiva, v. 21, n. 10, p. 3037-3047, 2016.

DUARTE, C. M. R. et al. Proteção social e política pública para populações vulneráveis: uma avaliação do Benefício de Prestação Continuada da Assistência Social - BPC no Brasil. Ciência \& Saúde Coletiva, v. 22, n. 11, p. 3515-3526, 2017. Disponível em: <https://doi.org/10.1590/1413-812320172211.22092017>. Acesso em: 08 jun. 2018.

DUARTE, C. M. R. et a. Regionalização e desenvolvimento humano: uma proposta de tipologia de Regiões de Saúde no Brasil. Caderno de Saúde Pública, v. 31, n. 6, p. 1163-1174, 2015.

FARIAS, N.; BUCHALLA, C. M. A classificação internacional de funcionalidade, incapacidade e saúde da organização mundial da saúde: conceitos, usos e perspectivas. Revista Brasileira de Epidemiologia, v. 8, n. 2, n. 187-93, 2005.

FURSTENAU, C. R.; WINK, JUNIOR, M. V. Medidas de extrema pobreza no Brasil, Grandes Regiões e Rio Grande do Sul: uma análise com os dados da PNAD 2011-14. 
Indicadores Econômicos, Porto Alegre, v. 43, n. 4, p. 79-90, 2016.

IBGE. Instituto Brasileiro de Geografia e Estatística, Diretoria de Pesquisas C de T e R. Pesquisa Nacional por Amostra de Domicílios Contínua - PNAD Contínua. Brasília, DF: IBGE, 2017. Disponível em:

<http://www.ibge.gov.br/home/estatistica/indicadores/trabalhoerendimento/pnad_contin ua/default_renda_percapita.shtm>. Acesso em: 30 abr. 2018.

JACOUD, L.; MESQUITA, A. C.; PAIVA, A. B. de. O Benefício de Prestação Continuada na Reforma da Previdência: contribuições para o debate. Instituto de Pesquisa Econômica Aplicada. Brasília: Rio de Janeiro: Ipea, 2017.

MACÊDO, A. C.; OLIVEIRA, L. C. Benefício de prestação continuada: perspectivas na avaliação médico-social. Revista Katálysis, v. 18, n. 1, p. 32-40, 2015.

PENALVA, J.; DINIZ, D.; MEDEIROS, M. O Benefício de Prestação Continuada no Supremo Tribunal Federal. Sociedade e Estado, v. 25, n. 1, p. 53-70, 2010.

SILVEIRA, F. G. et al. Deficiência e dependência no debate sobre a elegibilidade ao BPC. Nota Técnica n. 31, 2016. Disponível em:

<http://repositorio.ipea.gov.br/handle/11058/7338>. Acesso em: 30 abr. 2018.

WHO. WORLD HEALTH ORGANIZATION. Environment and health in developing countries: Priority risks and future trends, The Health and Environment Linkages Initiative (HELI). Disponível em:

<http://www.who.int/heli/risks/ehindevcoun/en/index1.html>. Acesso em: 30 abr. 2018.

XIMENES, J. M. Judicialização dos benefícios de prestação continuada e impactos simbólicos na cidadania. Pensar, v. 21, n. 2, p. 600-625, 2016. 